H. YANAMOTO

KODAI MATH. J.

8 (1985), 224-235

\title{
ON THE ELASTIC CLOSED PLANE CURVES
}

\author{
By HiRoshi YANAMOTO
}

\section{$\S 1$. Introduction.}

With respect to the total curvature of a closed curve $C$ of class $C^{2}$ in a 3dimensional Euclidean space $E^{3}$, we have the classical Fenchel inequality ([3] in 1929)

$$
\int_{C} k(s) d s \geqq 2 \pi,
$$

where $s$ denotes the arc length parameter of $C$ and $k(s)$ the curvature of $C$. If a closed curve $C$ is knotted in $E^{3}$, then the Fary inequality

$$
\int_{C} k(s) d s \geqq 4 \pi
$$

holds good (cf. Fary [2] and J. Milnor [5]).

If a closed curve $C$ is regarded as an elastic rod, then the bending energy $E(C)$ of the deflected curve $C$ from $k=0$ is given by (cf. [4], [8])

$$
E(C)=\frac{1}{2} \int_{C} k^{2}(s) d s .
$$

For any real number $t$, we get

$$
0 \leqq \int_{C}(k(s)-t)^{2} d s=\int_{C} k^{2}(s) d s-2 t \int_{C} k(s) d s+t^{2} \int_{C} d s .
$$

Then, from (1.1) we obtain

$$
E(C)=\frac{1}{2} \int_{C} k^{2}(s) d s \geqq 2 \pi^{2} / L,
$$

where $L$ is the length of the closed curve $C$. The equality holds good if and only if $C$ is a circle of radius $L / 2 \pi$ in the plane.

Concerning the inequality (1.4), I. Bives $([1]$, p. 283) showed the following:

Let $M$ be a circle of radius $r$, isometrically immersed into $E^{N}$. If $k$ denotes the curvature function, then

$$
\int_{N} k^{2}(s) d s \geqq 2 \pi / r
$$

Received June 5, 1984 
with equality iff $M$ is embedded as a circle. tional

The pourpose of this note is to study the variational problem of the func-

$$
E(C)=\frac{1}{2} \int_{C} k^{2}(s) d s
$$

under the condition $\int_{C} d s=L=$ constant. The equilibrium states of the elastic curves are the stationary points of the bending energy $E(C)$ with $L=$ constant. If the second variation of $E$ evaluated at some equilibrium state is positive definite, then the equilibrium state is called stable.

Thus we have the following questions (Bernoulli's problem).

$\left(Q_{1}\right)$ Find the closed curves on the plane $E^{2}$ for which the functional $E$ is stationary under the constant length.

$\left(Q_{2}\right)$ Investigate the elastic stability for these stationary curves.

We study the following classical Euler's theorem

THEOREM A. If $E(C)=\frac{1}{2} \int_{C} k^{2}(s) d s$ is critzcal for a closed plane curve with $L=$ constant, then the curve $C$ is either the plane circle $C_{n}$ (cf. Fig. 1) with the radius $L / 2 \pi n$ or the curve $D_{m}$ (cf. Fig. 2) which is congruent ot

$$
\left\{\begin{array}{l}
x(s)=\frac{2 p}{\sqrt{R}} \cos \phi, \\
y(s)=\frac{1}{\sqrt{R}} \int_{-\pi / 2}^{\phi}\left(2 \sqrt{1-p^{2} \sin ^{2} \phi}-\frac{1}{\sqrt{1-p^{2} \sin ^{2} \phi}}\right) d \phi,
\end{array}\right.
$$

where $\phi$ varies from $-\pi / 2$ to $3 \pi / 2$ and $R, p^{2}$ are given by

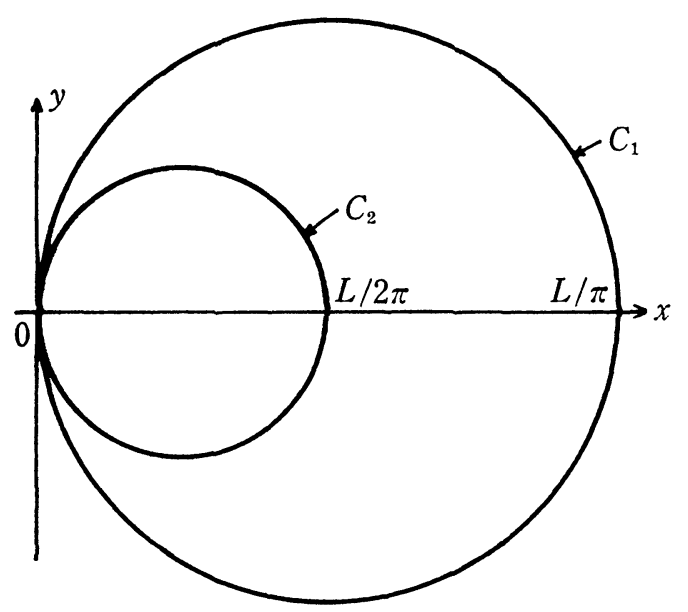

Fig. 1 


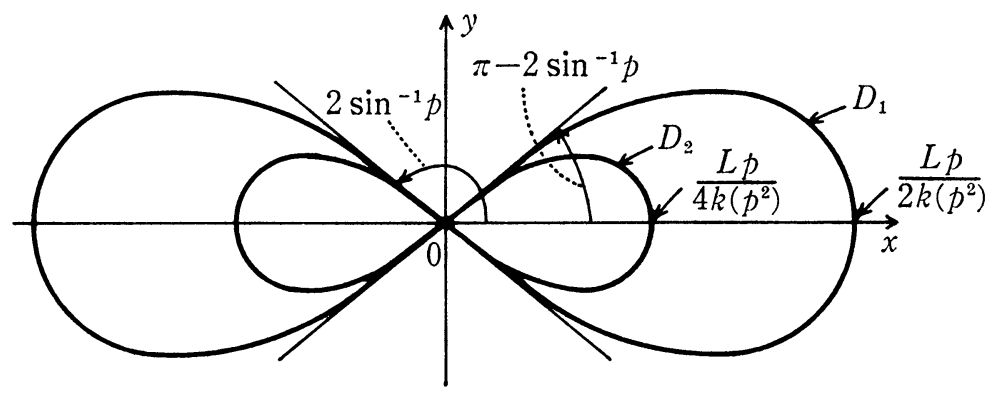

Fig. 2

$$
\begin{aligned}
& \sqrt{R}=\frac{4 m}{L} \int_{0}^{\pi / 2} \frac{1}{\sqrt{1-p^{2} \sin ^{2} \phi}} d \phi\left(=\frac{4 m}{L} K\left(p^{2}\right)\right), \\
& p^{2} ; 2 \int_{0}^{\pi / 2} \sqrt{1-p^{2} \sin ^{2} \phi} d \phi=\int_{0}^{\pi / 2} \frac{1}{\sqrt{1-p^{2} \sin ^{2} \phi}} d \phi .
\end{aligned}
$$

$p^{2}$ and $\sin ^{-1} p$ are in the intervals;

$$
0.82<p^{2}<0.83 \text { and } 1.13 \operatorname{Rad}<\sin ^{-1} p<1.15 \operatorname{Rad}
$$

For $C_{n}$ and $D_{m}$, the critical values are as follows:

and

$$
E\left(C_{n}\right)=2 \pi^{2} n^{2} / L, \quad E\left(D_{m}\right)=16 m^{2}\left(2 p^{2}-1\right) K^{2}\left(p^{2}\right) / L
$$

$$
E\left(C_{1}\right)=2 \pi^{2} / L<E\left(D_{1}\right)=16\left(2 p^{2}-1\right) K^{2}\left(p^{2}\right) / L<E\left(C_{2}\right)=8 \pi^{2} / L .
$$

\section{§2. Critical closed plane curves.}

Let $C:[0, L] \ni s \rightarrow(x(s), y(s)) \in E^{2}$ be a $C^{2}$ plane curve with arc length parameter $s$. Then the tangent vector $(d x / d s, d y / d s)$ to the curve is of unit length and satisfies the Frenet equation

$$
\frac{d^{2} x}{d s^{2}}=-k(s) \frac{d y}{d s}, \quad \frac{d^{2} y}{d s^{2}}=k(s) \frac{d x}{d s} .
$$

If $\theta(s)$ is the angle between the tangent $(d x / d s, d y / d s)$ and the positive $x$-axis, the curvature function $k(s)$ is given by

$$
k(s)=\frac{d \theta}{d s} .
$$

Assuming that $(x(0), y(0))=0$ (the origin in $\left.E^{2}\right),(x(s), y(s))$ is written by

$$
x(s)=\int_{0}^{s} \cos \theta(s) d s, \quad y(s)=\int_{0}^{s} \sin \theta(s) d s .
$$

Necessary and sufficient conditions for this curve $C$ to be closed are 
(a) $k(s)$ is periodic with period deviding $L$,

(b) $\theta(L)-\theta(0)$ is $2 \pi n$ ( $n=$ the rotation index of $C$ ),

(c) $x(L)=y(L)=0$.

Now we consider the variational problem with respect to $E(C)=\frac{1}{2} \int_{C} k^{2}(s) d s$ witn $L=$ constant. For an arbitrary variation $C_{\varepsilon}$ of $C$ such that

$$
C_{\varepsilon}: \theta_{\varepsilon}(s)=\theta(s)+\eta_{\varepsilon}(s) \quad\left(\eta_{\varepsilon}(0)=\eta_{\varepsilon}(L)=0\right),
$$

we get

$$
E\left(C_{\varepsilon}\right)=\frac{1}{2} \int_{C_{\varepsilon}}\left(k(s)+\frac{\partial \eta_{\varepsilon}(s)}{\partial s}\right)^{2} d s .
$$

Putting $\eta_{s}(s)=\varepsilon \eta(s)+\varepsilon^{2} h(s)+\left[\varepsilon^{3}\right]$, we see that

$$
\begin{aligned}
& E\left(C_{\varepsilon}\right)=\frac{1}{2} \int_{0}^{L}\left[k^{2}(s)+2 \varepsilon \frac{d \theta}{d s} \frac{d \eta}{d s}+\varepsilon^{2}\left\{\left(\frac{d \eta}{d s}\right)^{2}+2 \frac{d \theta}{d s} \frac{d h}{d s}\right\}\right] d s+\left[\varepsilon^{3}\right], \\
& \int_{C_{\varepsilon}} \cos \left(\theta(s)+\eta_{\varepsilon}(s)\right) d s=-\varepsilon \int_{0}^{L} \eta(s) \sin \theta(s) d s+\left[\varepsilon^{2}\right], \\
& \int_{C_{\varepsilon}} \sin \left(\theta(s)+\eta_{\varepsilon}(s)\right) d s=\varepsilon \int_{0}^{L} \eta(s) \cos \theta(s) d s+\left[\varepsilon^{2}\right] .
\end{aligned}
$$

If $C$ is critical, then we have

$$
0=\left.\frac{d E\left(C_{\varepsilon}\right)}{d \varepsilon}\right|_{\varepsilon=0}=\int_{0}^{L} \frac{d \theta}{d s} \frac{d \eta}{d s} d s=-\int_{0}^{L} \frac{d^{2} \theta}{d s^{2}} \eta(s) d s,
$$

for any $\eta(s)$ satisfying

$$
-\int_{0}^{L} \eta(s) \sin \theta(s) d s=\int_{0}^{L} \eta(s) \cos \theta(s) d s=0 .
$$

From this there exist two constants $\lambda$ and $\mu$ such that

$$
-\lambda \sin \theta+\mu \cos \theta=\frac{d^{2} \theta}{d s^{2}} \text {. }
$$

That is,

$$
-R \sin (\theta-\alpha)=\frac{d^{2} \theta}{d s^{2}}
$$

holds good, where we have put

and

$$
R=\sqrt{\lambda^{2}+\mu^{2}}
$$

$$
\alpha: R \cos \alpha=\lambda, \quad R \sin \alpha=\mu .
$$

In the case of $R=0$, we have 


$$
k(s)=\frac{d \theta}{d s}=\frac{2 \pi}{L} n \quad(n=1,2,3, \cdots) .
$$

This means geometrically that the closed plane curve $C$ is a circle $C_{n}$ of radius $L /(2 \pi n)$. The rotation index of $C_{n}$ is $n(n=1,2,3, \cdots)$.

In the case of $R>0$, multiplying (2.5) by $d \theta / d s$, we have

$$
d+R \cos (\theta-\alpha)=\frac{1}{2}\left(\frac{d \theta}{d s}\right)^{2},
$$

where $d$ is a constant of integration.

In the case of $-R<d<R$, putting $p=[(d+R) / 2 R]^{1 / 2}$, we obtain

$$
2 R\left\{p^{2}-\sin ^{2}\left(\frac{\theta-\alpha}{2}\right)\right\}=\frac{1}{2}\left(\frac{d \theta}{d s}\right)^{2}
$$

and hence

$$
k(s)=\frac{d \theta}{d s}= \pm 2 \sqrt{R} \sqrt{p^{2}-\sin ^{2}\left(\frac{\theta-\alpha}{2}\right)},
$$

where $p$ and $\theta$ satisfy the following:

$$
0<p<1, \quad-p \leqq \sin \left(\frac{\theta-\alpha}{2}\right) \leqq p .
$$

We put

$$
\theta(0)=\alpha-2 \sin ^{-1} p \quad\left(0<\sin ^{-1} p<\pi / 2\right) .
$$

Then, taking account of the conditions (a) and (b), (2.7) may be written in the form

$$
s=\frac{1}{2 \sqrt{R}} \int_{d-2 \sin ^{-1} p}^{\theta} \frac{1}{\sqrt{p^{2}-\sin ^{2}\left(\frac{\theta-\alpha}{2}\right)}} d \theta
$$

where $s$ and $\theta$ run on

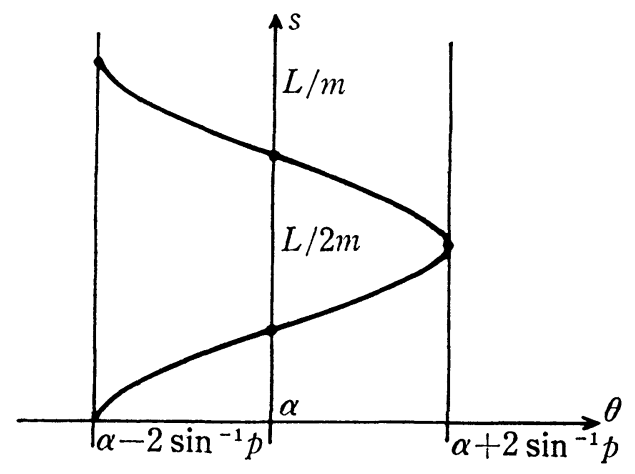

Fig. 3 


$$
0 \leqq s \leqq \frac{L}{2 m}, \quad \alpha-2 \sin ^{-1} p \leqq \theta \leqq \alpha+2 \sin ^{-1} p .
$$

The integration of (2.9) can be simplified by using

$$
\begin{aligned}
& \sin \left(\frac{\theta-\alpha}{2}\right)=p \sin \phi, \\
& \frac{\theta-\alpha}{2}=\sin ^{-1}(p \sin \phi) .
\end{aligned}
$$

It is seen from $(2.11)^{\prime}$ that when $\theta$ varies from $\alpha-2 \sin ^{-1} p$ to $\alpha+2 \sin ^{-1} p$ the quantity $\phi$ varies from $-\pi / 2$ to $\pi / 2$.

On the other hand, from (2.7) and (2.11), we obtain

$$
\left(\frac{d \theta}{d s}\right)^{2}\left\{\left(\frac{d \phi}{d s}\right)^{2}-R\left(1-p^{2} \sin ^{2} \phi\right)\right\}=0
$$

from which

$$
\frac{d \phi}{d s}=\sqrt{R} \sqrt{1-p^{2} \sin ^{2} \phi} \quad\left(\sin ^{2} \phi \neq 1\right)
$$

Hereby, we have

$$
s=\frac{1}{\sqrt{R}} \int_{-\pi / 2}^{\phi} \frac{1}{\sqrt{1-p^{2} \sin ^{2} \phi}} d \phi,
$$

where $s$ and $\phi$ run on $0 \leqq s \leqq L / m$ and $-\pi / 2 \leqq \phi \leqq 3 \pi / 2$. Putting

$$
K\left(p^{2}\right)=\int_{0}^{\pi / 2} \frac{1}{\sqrt{1-p^{2} \sin ^{2} \phi}} d \phi \quad(0<p<1),
$$

we obtain

$$
L / m=\frac{4}{\sqrt{R}} K\left(p^{2}\right) .
$$

$K\left(p^{2}\right)$ is known as the complete elliptic integral of the first kind. Next we must check whether the condition (c) is satisfied or not for the curve given by

$$
\left\{\begin{array}{l}
\theta=\alpha+2 \sin ^{-1}(p \sin \phi), \quad-\pi / 2 \leqq \phi \leqq 3 \pi / 2, \\
s=\frac{1}{\sqrt{R}} \int_{-\pi / 2}^{\phi} \frac{1}{\sqrt{1-p^{2} \sin ^{2} \phi}} d \phi .
\end{array}\right.
$$

By (2.17), we have

$$
\begin{aligned}
\int_{0}^{L} \cos \theta(s) d s & =m \int_{0}^{L / m} \cos \theta(s) d s \\
& =\frac{m}{\sqrt{R}} \int_{-\pi / 2}^{3 \pi / 2} \frac{\cos \left\{\alpha+2 \sin ^{-1}(p \sin \phi)\right\}}{\sqrt{1-p^{2} \sin ^{2} \phi}} d \phi,
\end{aligned}
$$

and one for $\sin \theta(s)$. In view of 


$$
\begin{aligned}
& \cos \left\{\alpha+2 \sin ^{-1}(p \sin \phi)\right\} \\
& =(\cos \alpha)\left(1-2 p^{2} \sin ^{2} \phi\right)-2 p \sin \alpha \sin \phi \sqrt{1-p^{2} \sin ^{2} \phi},
\end{aligned}
$$

etc., we get

$$
\begin{aligned}
& \int_{0}^{L} \cos \theta(s) d s=\frac{m \cos \alpha}{\sqrt{R}} \int_{-\pi / 2}^{3 \pi / 2} \frac{1-2 p^{2} \sin ^{2} \phi}{\sqrt{1-p^{2} \sin ^{2} \phi}} d \phi \\
& \int_{0}^{L} \sin \theta(s) d s=\frac{m \sin \alpha}{\sqrt{R}} \int_{-\pi / 2}^{3 \pi / 2} \frac{1-2 p^{2} \sin ^{2} \phi}{\sqrt{1-p^{2} \sin ^{2} \phi}} d \phi .
\end{aligned}
$$

Therefore, the condition (c) is equivalent to

$$
2 \int_{0}^{\pi / 2} \sqrt{1-p^{2} \sin ^{2} \phi} d \phi=\int_{0}^{\pi / 2} \frac{1}{\sqrt{1-p^{2} \sin ^{2} \phi}} d \phi
$$

Putting

$$
E\left(p^{2}\right)=\int_{0}^{\pi / 2} \sqrt{1-p^{2} \sin ^{2} \phi} d \phi
$$

we have

$$
2 E\left(p^{2}\right)=K\left(p^{2}\right), \quad p^{2}=\frac{d+R}{2 R} .
$$

$E\left(p^{2}\right)$ is known as the complete elliptic integral of the second kind. Using the Iwanami Math. dictionary ([7], second edition, p. 974, Fig. 12), we get

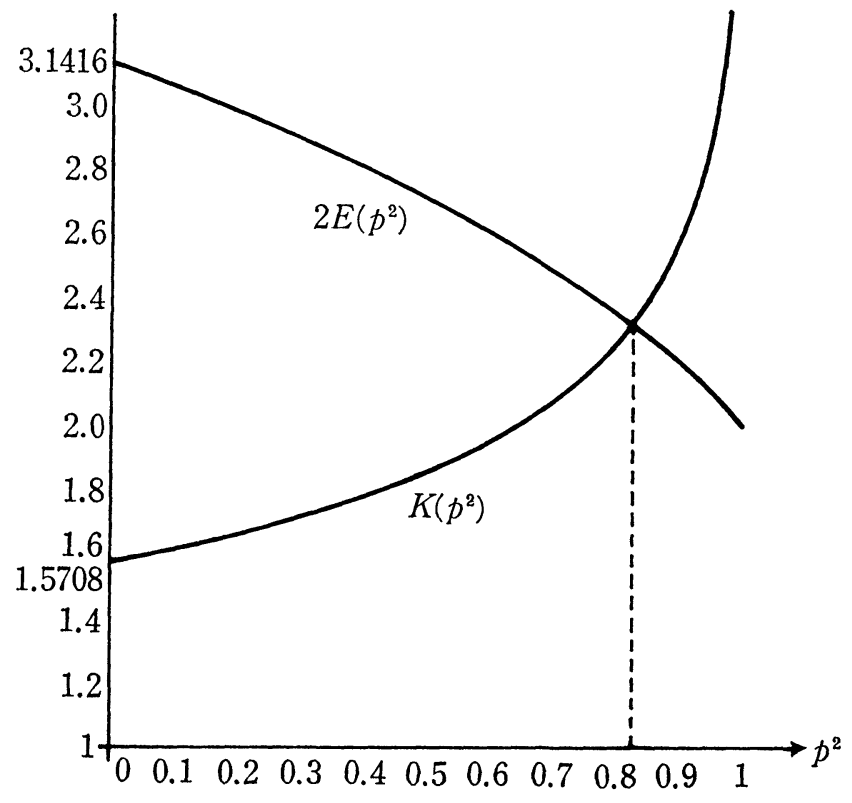

Fig. 4 
We see that there is the constant $p^{2}$ in the interval

$$
0.82<p^{2}<0.83
$$

Hence we have

$$
129^{\circ} 29^{\prime} 19^{\prime \prime}<2 \sin ^{-1} p<131^{\circ} 46^{\prime} 49^{\prime \prime} .
$$

We see that $(x(s), y(s))$ is given by

$$
\left\{\begin{array}{l}
x(s)=\frac{1}{\sqrt{R}}\left\{2 p \sin \alpha \cos \phi+\cos \alpha \int_{-\pi / 2}^{\phi}\left(2 \sqrt{1-p^{2} \sin ^{2} \phi}-\frac{1}{\sqrt{1-p^{2} \sin ^{2} \phi}}\right) d \phi\right\}, \\
y(s)=\frac{1}{\sqrt{R}}\left\{-2 p \cos \alpha \sin \phi+\sin \alpha \int_{-\pi / 2}^{\phi}\left(2 \sqrt{1-p^{2} \sin ^{2} \phi}-\frac{1}{\sqrt{1-p^{2} \sin ^{2} \phi}}\right) d \phi\right\},
\end{array}\right.
$$

\begin{tabular}{|c|c|c|c|c|c|c|}
\hline$s$ & 0 & $\cdots$ & $L /(4 m)$ & $\cdots, \quad L /(2 m)$ & $3 L /(4 m)$ & $L / m$ \\
\hline$\phi$ & $-\pi / 2$ & & 0 & $\pi / 2$ & $\pi$ & $3 \pi / 2$ \\
\hline$\theta$ & $\alpha-2 \sin ^{-1} p^{2}$ & & $\alpha$ & $\alpha+2 \sin ^{-1} p^{\prime}$ & $\alpha$ & $\alpha-2 \sin ^{-1} p$ \\
\hline$k(s)$ & 0 & + & $2 \sqrt{R} p$ & + & $-2 \sqrt{R} p$ & 0 \\
\hline$x(s)$ & 0 & & $2 p \sin \alpha / \sqrt{R}$ & 0 & $-2 p \sin \alpha / \sqrt{R}$ & 0 \\
\hline$y(s)$ & 0 & 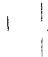 & $-2 p \cos \alpha / \sqrt{R}$ & 0 & $2 p \cos \alpha / \sqrt{R}$ & 0 \\
\hline
\end{tabular}

from which we get the following table.

The closed plane curve $C$ may be drawn as follows (cf. Fig. 5).

Remark 1. In particular, for $\alpha=\pi / 2$, we get

$$
\left\{\begin{array}{l}
x(s)=\frac{2 p}{\sqrt{R}} \cos \phi \\
y(s)=\frac{1}{\sqrt{R}} \int_{-\pi / 2}^{\phi}\left(2 \sqrt{1-p^{2} \sin ^{2} \phi}-\frac{1}{\sqrt{1-p^{2} \sin ^{2} \phi}}\right) d \phi .
\end{array}\right.
$$

Remark 2. For $D_{m}$, we get

$$
\begin{aligned}
E\left(D_{m}\right) & =\frac{1}{2} \int_{0}^{L}(d \theta / d s)^{2} d s=\frac{m}{2} \int_{-\pi / 2}^{3 \pi / 2} \frac{d \phi}{d s}\left(\frac{d \theta}{d \phi}\right)^{2} d \phi \\
& =8 m \sqrt{R} \int_{0}^{\pi / 2}\left\{\sqrt{1-p^{2} \sin ^{2} \phi}-\frac{1-p^{2}}{\sqrt{1-p^{2} \sin ^{2} \phi}}\right\} d \phi \\
& =\frac{16 m^{2}}{L}\left(2 p^{2}-1\right) K^{2}\left(p^{2}\right) .
\end{aligned}
$$

For instance we have 


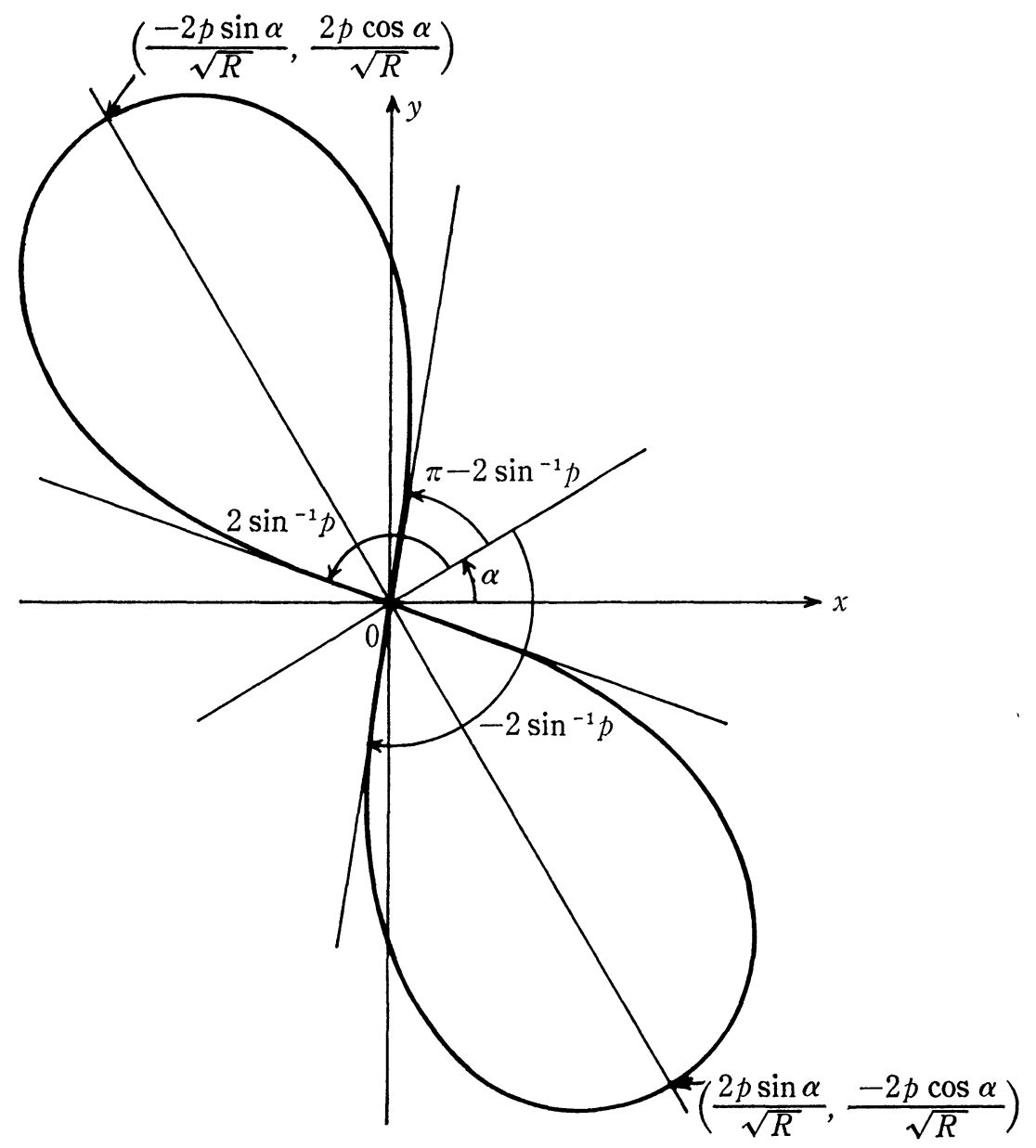

Fig. 5

$$
\begin{aligned}
E\left(C_{1}\right)=2 \pi^{2} / L=19.74 / L<E\left(D_{1}\right) & =\frac{16}{L}\left(2 p^{2}-1\right) K^{2}\left(p^{2}\right) \doteqdot 54.42 / L \\
& <E\left(C_{2}\right)=\frac{8 \pi^{2}}{L} \doteqdot 78.96 / L
\end{aligned}
$$

Let us now turn to the case $d=R$ or $d>R$. In the case of $d=R$, by (2.7) and $\theta(0)=\alpha$ we get

$$
k(s)=d \theta / d s=2 \sqrt{R} \cos \left(\frac{\theta-\alpha}{2}\right),
$$

from which 


$$
s=\int_{\alpha}^{\theta} \frac{1}{2 \sqrt{R} \cos \left(\frac{\theta-\alpha}{2}\right)} d \theta=\frac{1}{\sqrt{R}} \log \left|\tan \left(\frac{\pi}{4}+\frac{\theta-\alpha}{4}\right)\right| .
$$

Thus we obtain

$$
e^{\sqrt{ } R} s=\tan \left(\frac{\pi}{4}+\frac{\theta-\alpha}{4}\right) .
$$

It requires the infinite arc length to obtain $\theta=\pi+\alpha$. Therefore, the case of $d=R$ does not occur.

In the case of $d>R$, putting

$$
q=\sqrt{2 R /(d+R)} \quad(0<q<1),
$$

we get

$$
k(s)=d \theta / d s=\sqrt{2(d+R)} \sqrt{1-q^{2} \sin ^{2}\left(\frac{\theta-\alpha}{2}\right)} .
$$

Suppose now that $\theta(0)=\alpha$. Then we have the following (cf. Fig. 6) :

$$
s=\frac{1}{\sqrt{2(d+R)}} \int_{\alpha}^{\theta} \frac{d \theta}{\sqrt{1-q^{2} \sin ^{2}\left(\frac{\theta-\alpha}{2}\right)}}\left(=\int_{\alpha}^{\theta} \frac{d \theta}{\sqrt{2\{d+R \cos (\theta-\alpha)\}}}\right) .
$$

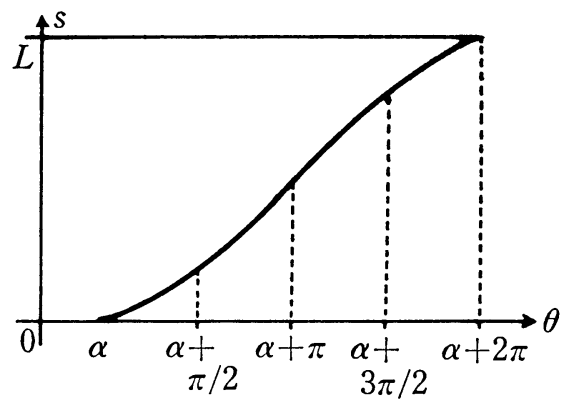

Fig. 6

Hence we obtain

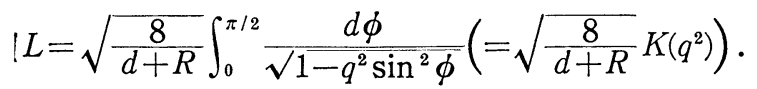

Let us now check whether the condition (c) is satisfied or not. By means of

$$
\begin{aligned}
& \int_{0}^{L} \cos \theta(s) d s=\frac{2}{\sqrt{2(d+R)}} \int_{0}^{\pi} \frac{\cos (\alpha+2 \phi)}{\sqrt{1-q^{2} \sin ^{2} \phi}} d \phi, \\
& \int_{0}^{L} \sin \theta(s) d s=\frac{2}{\sqrt{2(d+R)}} \int_{0}^{\pi} \frac{\sin (\alpha+2 \phi)}{\sqrt{1-q^{2} \sin ^{2} \phi}} d \phi,
\end{aligned}
$$


the condition (c) is equivalent to

$$
\int_{0}^{\pi} \frac{\cos 2 \phi}{\sqrt{ } 1-q^{2} \sin ^{2} \phi} d \phi=0, \quad \int_{0}^{\pi} \frac{\sin 2 \phi}{\sqrt{1-q^{2} \sin ^{2} \phi}} d \phi=0 \text {. }
$$

The first equation reduces to

$$
2 \int_{0}^{\pi / 2} \sqrt{1-q^{2} \sin ^{2} \phi} d \phi=\left(2-q^{2}\right) \int_{0}^{\pi / 2} \frac{1}{\sqrt{1-q^{2} \sin ^{2} \phi}} d \phi,
$$

that is,

$$
2 E\left(q^{2}\right)=\left(2-q^{2}\right) K\left(q^{2}\right), \quad 0<q^{2}<1 .
$$

However, we can verify that $2 E\left(q^{2}\right)<\left(2-q^{2}\right) K\left(q^{2}\right)$. In fact, $\left(2-q^{2}\right) K\left(q^{2}\right)-2 E\left(q^{2}\right)$ $=\pi q^{4} / 16+\left(q^{6}\right)$ for $q^{2} \doteqdot 0$, and we get the following figure (cf. Fig. 7).

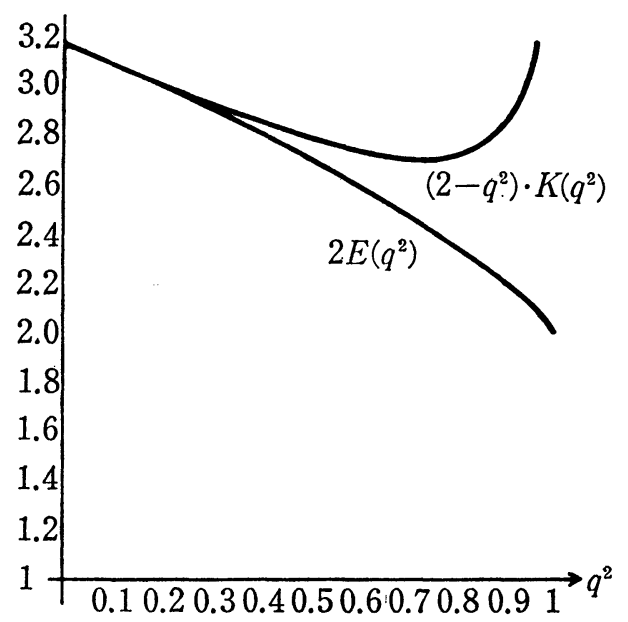

Fig. 7

Hence the curve $C$ given by (2.22) does not satisfy the condition (c), that is, the curve is not closed.

Summarizing the results obtained above, we get the theorem $\mathrm{A}$ in the introduction.

\section{BIBLIOGRAPHY}

[1] Bivens, I., Codazzi Tensors and Reducible submanifolds, Trans. Amer. Math. Soc., 268 (1981), 231-246.

[2] FARY, M.I., Sur la Courbure Totale d'une Courbe Gauche Faisant un Noeund, Bull. Soc. Math. France, 77 (1949), 128-138.

[3] Fenchel, W., Über Krümmung und Windung geschlossener Raumkurven, Math. Ann., 101 (1929), 238-252.

[4] KUBOI, F. AND Y. EzAwA, The various characteristic and its constraction of the critical deflected curves with respect to the bending energy (in Japanese, Jyôhō 
Syôri Gakkai, Dai 22 kai (1981) Zenkokutaikai), 2H-3, 987-988.

[5] Milnor, J., On the total curvature of knots, Ann. Math., 52 (1950), 248-257.

[6] SAGAN, H., Introduction to the calculus of variations, Int. Ser. pure Appl. Math., McGraw-Hill, 1969.

[7] Iwanami Mathematical Dictionary, Second Ed. (1968), Iwanami Shoten, in Japan.

[8] Griffiths, P.A., Exterior differential systems and the calculus of variations, Birkhäuser, 1983.

\section{Addition}

$[1]^{\prime}$ Antman, S.S., The influence of elasticity on analysis: modern developments, Bull. Amer. Math. Soc., 9 (1983), 267-291.

$[2]^{\prime}$ GAGE, M.E., An isoperimetric inequality with applications to curve shortening, Duke Math. J., Vol. 50, No. 4, (1983), 1225-1229.

$[3]^{\prime} \longrightarrow$, Curve shortening makes convex curves circular, Invent. Math., 76 (1984), 357-364.

$[4]^{\prime}$ TRUesdell, C., The influence of elasticity on analysis: the classic heritage, Bull. Amer. Math. Soc., 9 (1983), 293-310.

Department of Mathematics

School of Liberal ARts AND Sciences

Iwate Medical University 